\title{
Synthesis of Adaptive Gain Robust Output Feedback Controllers for a Class of Lipschitz Nonlinear Systems with Unknown Upper Bound of Uncertainty
}

\author{
Hidetoshi Oya ${ }^{1}$ and Kojiro Hagino ${ }^{2}$ \\ ${ }^{1}$ Institute of Technology and Science, The University of Tokushima, 2-1 Minamijosanjima, Tokushima 770-8506, Japan \\ ${ }^{2}$ Department of Systems Engineering, The University of Electro-Communications, 1-5-1 Chofugaoka, Chofu, Tokyo 182-8585, Japan
}

Correspondence should be addressed to Hidetoshi Oya, hide-o@ee.tokushima-u.ac.jp

Received 9 May 2012; Accepted 6 July 2012

Academic Editor: Zhiyong Chen

Copyright ( $) 2012$ H. Oya and K. Hagino. This is an open access article distributed under the Creative Commons Attribution License, which permits unrestricted use, distribution, and reproduction in any medium, provided the original work is properly cited.

\begin{abstract}
We propose a new adaptive gain robust output feedback controller for a class of the Lipschitz nonlinear systems with unknown upper bound of uncertainty. The proposed adaptive gain robust output feedback controller is designed so as to reduce the effect of uncertainties and Lipschitz nonlinearities. In this paper, we show that sufficient conditions for the existence of the proposed adaptive gain robust output feedback controller are reduced to LMI conditions. Finally, the effectiveness of the proposed robust output feedback controller is demonstrated by numerical simulations.
\end{abstract}

\section{Introduction}

Robustness of control systems to uncertainties has always been the central issue in feedback control, and therefore the problems of stability analysis and stabilization for uncertain systems have received much attention for a long time (e.g., $[1,2]$ and references therein). In particular, there are lots of existing results for state feedback robust control such as quadratic stabilizing control and $\mathscr{H}^{\infty}$ control (see $[3,4]$ and references therein). Besides, some design methods of variable gain robust state feedback controllers for uncertain systems have been suggested (e.g., [5-8]). Yamamoto and Yamauchi [5] proposed a design method of a robust controller with the ability to adjust control performances adaptively. In [6], an adaptive robust controller with adaptation mechanism has been presented and the adaptive robust controller is tuned on-line based on the information about parameter uncertainties. Besides, we have proposed robust controllers with adaptive compensation inputs $[7,8]$. These controllers consist of a fixed gain controller and a variable gain one, and the variable gain controller is tuned by updating laws.

However, not all the states are measurable in practical systems because of technical, physical, and/or economic reasons. Therefore, the control strategies via observer-based robust controllers (e.g., $[9,10])$ or robust output feedback one (e.g., [11-13]), which is of interest in this paper, have also been well studied. For robust output feedback controllers, Moheimani and Petersen [11] have presented a set of crosscoupled algebraic Riccati equations and algebraic Lyapunov equations. Geromel et al. [12] and Iwasaki et al. [13] adopted linear matrix inequality (LMI) approaches to design static output feedback controllers based on a set of the Lyapunov inequalities coupled by the constraint that one Lyapunov matrix is the inverse of another. Additionally the work of Matsuoka and Hagino [14] has presented an observer-based variable gain controller for a class of linear systems with uncertainties of which upper bounds are unknown, and we have also proposed an adaptive robust output feedback controller for a class of linear systems with uncertainties [15].

By the way, in recent years, there have been increasing attention for the problem of global stabilization of nonlinear systems via output feedback control (e.g., [16-18]). In the work of Mazenc et al. [16], it was presented through counter examples that some extra growth conditions on the unmeasurable states of the controlled system are usually necessary for the global stabilization of nonlinear systems 
via output feedback. Additionally, finite-time method and homogeneous domination approach for the output feedback control problem have also been proposed (e.g., [19-21]). Polendo and Qian [20] have suggested output feedback controllers for a class of uncertain nonlinear systems via homogeneous domination approach, and Li and Qian [21] adopted the concept of finite-time stabilization so as to design a dynamic output feedback controller for a class of continuous but nonsmooth nonlinear systems. Besides, some results have focused on considering a selective class of nonlinear systems by placing some structural constraints on the nonlinearities in order to derive output feedback control. The nonlinear systems whose nonlinearity is in a triangular form are considered in [22]. In the work of Choi and Lim [23], a solution to the output feedback stabilization problem for a class of single-input single-output Lipschitz nonlinear systems and the nonlinearity characterization function (NCF) concept has been presented. However, in the existing results, the design parameters are determined by trial and error, and uncertainties in the system dynamics have not been considered.

From these viewpoints on the basis of our results $[15,24]$, we propose a new adaptive gain robust output feedback controller for a class of uncertain Lipschitz nonlinear systems. The uncertainties and the nonlinearities under consideration supposed to satisfy the matching condition, and the perturbation region of uncertainty is bounded, but its upper bound is unknown. Besides, the proposed adaptive gain robust output feedback controller consists of a fixed gain controller, a variable gain one, and an adjustable parameter tuned by updating laws. The fixed gain controller is determined by using the nominal system, and the variable gain controller and the adjustable parameter are designed so as to reduce the effect of both uncertainties and nonlinearities. In this paper, we show that sufficient conditions for the existence of the proposed adaptive gain robust output feedback controller are reduced to LMI conditions.

This paper is organized as follows. In Section 2, we introduce the class of uncertain Lipschitz nonlinear systems under consideration. Section 3 contains the main results. The design method of the adaptive robust output feedback controller for the uncertain Lipschitz nonlinear systems is presented. Finally, numerical examples are included to illustrate the results developed in this paper.

\section{Preliminaries}

In this section, we show notations and useful and wellknown lemmas which are used in this paper.

In the sequel, we use the following notation. For a matrix $\mathcal{A}$, the transpose of matrix $\mathcal{A}$ and the inverse of one are denoted by $\mathcal{A}^{T}$ and $\mathcal{A}^{-1}$, respectively, and $\operatorname{rank}\{\mathcal{A}\}$ represents the rank of the matrix $\mathcal{A}$. Also, $H_{e}\{\mathcal{A}\}$ and $I_{n}$ represent $\mathcal{A}+\mathcal{A}^{T}$ and $n$-dimensional identity matrix, respectively, and $\operatorname{vec}(\mathscr{A})$ denotes the column vector of the matrix $\mathcal{A}$; that is, the operator "vec" vectorizes a matrix by stacking its columns. The notation $\operatorname{diag}\left(\mathscr{A}_{1}, \ldots, \mathcal{A}_{N}\right)$ denotes a block diagonal matrix composed of matrices $\mathcal{A}_{i}$ for $i=1, \ldots, N$. For real symmetric matrices $\mathcal{A}$ and $\mathcal{B}$,
$\mathcal{A}>\mathscr{B}$ (resp., $\mathcal{A} \geq \mathscr{B}$ ) means that $\mathcal{A}-\mathscr{B}$ is positive (resp., nonnegative) definite matrix. For a vector $\alpha \in \mathbb{R}^{n}$, $\|\alpha\|$ denotes standard Euclidian norm, and for a matrix $\mathcal{A}$, $\|\mathcal{A}\|$ represents its induced norm. The symbols " $\triangleq$ " and “ $\star$ ” mean equality by definition and symmetric blocks in matrix inequalities, respectively. Besides, for a symmetric matrix $\mathcal{P}$, $\lambda_{\min }\{\mathcal{P}\}$ (resp., $\lambda_{\max }\{\mathcal{P}\}$ ) represents the minimal eigenvalue (resp. maximal eigenvalue). It is well-known that for any symmetric matrix $\mathcal{P} \in \mathbb{R}^{n \times n}$, eigenvalues of $\mathcal{P} \in \mathbb{R}^{n \times n}$ are real number [25].

Furthermore, the following well-known lemmas are used in this paper.

Lemma 1. For arbitrary vectors $\lambda$ and $\xi$ and the matrices $g$ and $\mathscr{H}$ which have appropriate dimensions, the following relation holds:

$$
\begin{aligned}
H_{e}\left\{\lambda^{T} g \Delta(t) \mathscr{H} \xi\right\} & \leq 2\left\|g^{T} \lambda\right\|\|\Delta(t)\|\|\mathscr{H} \xi\| \\
& \leq 2 \vartheta^{*}\left\|g^{T} \lambda\right\|\|\mathscr{H} \xi\|,
\end{aligned}
$$

where $\Delta(t) \in \mathbb{R}^{p \times q}$ is a time-varying unknown matrix satisfying $\|\Delta(t)\| \leq \mathfrak{\vartheta}^{*}$.

Proof. The above relation can be easily obtained by Schwartz's inequality [25].

Lemma 2 (Schur complement). For a given constant real symmetric matrix $\Xi$, the following items are equivalent:
(i) $\Xi=\left(\begin{array}{ll}\Xi_{11} & \Xi_{12} \\ \Xi_{12}^{T} & \Xi_{22}\end{array}\right)>0$,
(ii) $\Xi_{11}>0$ and $\Xi_{22}-\Xi_{12}^{T} \Xi_{11}^{-1} \Xi_{12}>0$,
(iii) $\Xi_{22}>0$ and $\Xi_{11}-\Xi_{12} \Xi_{22}^{-1} \Xi_{12}^{T}>0$.

Proof. See Boyd et al. [26].

Lemma 3 ( $\&$-procedure). Let $\mathcal{F}(x)$ and $\mathcal{g}(x)$ be two arbitrary quadratic forms over $\mathbb{R}^{n}$. Then $\mathcal{F}(x)<0$ for all $x \in \mathbb{R}^{n}$ satisfying $G(x) \leq 0$ if and only if there exists a nonnegative scalar $\tau$ such that

$$
\mathcal{F}(x)-\tau g(x) \leq 0 \quad \text { for } \forall x \in \mathbb{R}^{n} .
$$

Proof. See Boyd et al. [26].

Lemma 4 (Barbalat's lemma). Let $\phi: \mathbb{R} \rightarrow \mathbb{R}$ be a uniformly continuous function on $[0, \infty)$. Suppose that $\lim _{t \rightarrow \infty} \int_{0}^{t} \phi(\tau) d \tau$ exists and is finite. Then

$$
\phi(t) \longrightarrow 0 \text { as } t \longrightarrow \infty \text {. }
$$

Proof. See Khalil [27].

\section{Problem Formulation}

Consider the uncertain Lipschitz nonlinear system described by the following state equation:

$$
\begin{gathered}
\frac{d}{d t} x(t)=(A+B \Delta(t) E) x(t)+B u(t)+\delta(x, t) \\
y(t)=C x(t)
\end{gathered}
$$


where $x(t) \in \mathbb{R}^{n}, u(t) \in \mathbb{R}^{m}$, and $y(t) \in \mathbb{R}^{l}$ are the vectors of the state, the control input, and the measured output, respectively. In (4), the matrices $A, B$, and $C$ are the nominal values of system parameters, and the matrix $\Delta(t) \in \mathbb{R}^{p \times q}$ denotes unknown time-varying parameters which satisfy

$$
\|\Delta(t)\| \leq \vartheta^{*},
$$

where the upper bound $\vartheta^{*}$ is bounded, but it is unknown. Additionally in this paper, we assume that the nonlinear term $\delta(x, t) \in \mathbb{R}^{n}$ in (4) is given by

$$
\delta(x, t)=B \xi(x, t),
$$

and for the function $\xi: \mathbb{R}^{n} \times R \rightarrow \mathbb{R}^{m}$, there exists a known positive constant scalar $\chi^{*}$ such that for all $x_{1}, x_{2} \in \mathbb{R}^{n}$

$$
\left\|\xi\left(x_{1}, t\right)-\xi\left(x_{2}, t\right)\right\| \leq \chi^{*}\left\|x_{1}-x_{2}\right\| .
$$

Note that since not all the states are measurable, the nonlinear term $\delta(x, t)$ is unknown. Besides, we introduce the following assumption for the system parameters $[15,24]$ :

$$
B^{T}=\mathcal{T} C,
$$

where $\mathcal{T} \in \mathbb{R}^{m \times l}$ is a known constant matrix.

The nominal system, ignoring unknown parameters and nonlinearities in (4), is given by

$$
\begin{gathered}
\frac{d}{d t} \bar{x}(t)=A \bar{x}(t)+B \bar{u}(t), \\
\bar{y}(t)=C \bar{x}(t),
\end{gathered}
$$

and it is supposed to be stabilizable via static output feedback control. Namely, there exists a static output feedback stabilizing control $\bar{u}(t)=K \bar{y}(t)$ (i.e., a fixed gain matrix $K \in \mathbb{R}^{m \times l}$ ). In other words since the nominal system of (9) is stabilizable via static output feedback control, the matrix $A_{K} \triangleq A+B K C$ is asymptotically stable. Note that the output feedback gain matrix $K \in \mathbb{R}^{m \times l}$ is designed by using the existing results (e.g., $[28,29])$. Besides, in this paper, we consider the following target model so as to generate the desirable trajectory:

$$
\begin{gathered}
\frac{d}{d t} x_{t}(t)=A x_{t}(t)+B u_{t}(t)+\delta\left(x_{t}, t\right), \\
y_{t}(t)=C x_{t}(t) .
\end{gathered}
$$

In order to generate the desirable trajectory for the uncertain system of (4), we select the control input for the target model such as $u_{t}(t)=K_{\mathscr{L} Q} x_{t}(t)-\xi\left(x_{t}, t\right)$ where $K_{\mathscr{L} Q} \in \mathbb{R}^{m \times n}$ is determined by adopting the standard LQ problem. Namely, by using the solution of the algebraic Riccati equation $H_{e}\left\{A^{T} \mathcal{X}_{t}\right\}-\mathcal{X}_{t} B \mathcal{R}_{t}^{-1} B^{T} \mathcal{X}_{t}+\mathcal{Q}_{t}=0$, the gain matrix $K_{\mathscr{L} Q}$ is determined as $K_{\mathcal{L Q}}=-\mathcal{R}_{t}^{-1} B^{T} \mathcal{X}_{t}$. Of course, some other design methods can also be utilized. Note that the target model with the control input $u_{t}(t)=K_{\mathcal{L} Q} x_{t}(t)-\xi\left(x_{t}, t\right)$ can be written as the following form:

$$
\begin{gathered}
\frac{d}{d t} x_{t}(t)=\left(A+B K_{\mathcal{L Q}}\right) x_{t}(t), \\
y_{t}(t)=C x_{t}(t) .
\end{gathered}
$$

Now on the basis of the works of Oya and Hagino ([15, 24]), by introducing the error vectors $e(t) \triangleq x(t)-x_{t}(t)$ and $e_{y}(t) \triangleq y(t)-y_{t}(t)$, we consider the following control input for the uncertain Lipschitz nonlinear system of (4):

$$
u(t) \triangleq K_{y}(t)+K_{\mathscr{L} Q} x_{t}(t)-\xi\left(x_{t}, t\right)+\psi\left(e_{y}, x_{t}, \hat{\theta}, t\right) .
$$

In (12), $\psi\left(e_{y}, x_{t}, \hat{\theta}, t\right) \in \mathbb{R}^{m}$ is an adaptive compensation input where $\hat{\theta}(t) \in \mathbb{R}^{1}$ is an adjustable parameter. Then one can see from (4), (6), and (10)-(12) that the following uncertain error system with nonlinear terms can be derived:

$$
\begin{aligned}
& \frac{d}{d t} e(t)= A_{K} e(t)+B \Delta(t) E x(t) \\
&+B\left(\xi(x, t)-\xi\left(x_{t}, t\right)\right)+B \psi\left(e_{y}, x_{t}, \hat{\theta}, t\right), \\
& e_{y}(t)=C e(t) .
\end{aligned}
$$

From the above, our control objective is to design the adaptive gain robust output feedback controller which achieves not only robust stability for the uncertain Lipschitz nonlinear system of (4) but also satisfactory transient behavior as closely as possible to desired trajectory generated by the target model. That is to derive the adaptive compensation input $\psi\left(e_{y}, x_{t}, \hat{\theta}, t\right) \in \mathbb{R}^{m}$ which stabilizes the uncertain nonlinear error system of (13).

\section{Main Results}

In this section, we show an LMI-based design method of the adaptive gain robust output feedback controller for the uncertain Lipschitz nonlinear system of (4). The following theorem gives an LMI-based design method of an adaptive gain robust output feedback controller.

Theorem 5. Consider the uncertain nonlinear error system of (13) with the adaptive compensation input $\psi\left(e_{y}, x_{t}, \hat{\theta}, t\right) \in$ $\mathbb{R}^{m}$.

If there exist symmetric positive definite matrices $\& \in$ $\mathbb{R}^{n \times n}, \Xi \in \mathbb{R}^{l \times l}$, and $\Psi \in \mathbb{R}^{l \times l}$ and the positive scalars $\gamma_{1}, \gamma_{2}$, and $\epsilon$ satisfying the LMIs:

$$
\begin{gathered}
H_{e}\left\{\delta A_{K}\right\}+\gamma_{1} E^{T} E+\epsilon\left(\chi^{*}\right)^{2} I_{n} \leq-\mathcal{Q}, \\
C^{T} \Xi C-H_{e}\left\{\delta C^{T} \mathcal{T}^{T} \mathcal{T} C\right\} \leq 0, \\
\left(\begin{array}{ccc}
-C^{T} \Psi_{\gamma} C & \delta C^{T} \mathcal{T} & 8 C^{T} \mathcal{T} \\
\star & -\gamma_{1} I_{m} & 0 \\
\star & \star & -\gamma_{2} I_{m}
\end{array}\right) \leq 0, \\
\left(\begin{array}{ccc}
-C^{T} \Psi_{\epsilon} C & s C^{T} \mathcal{T}^{T} \\
\star & -\epsilon I_{m}
\end{array}\right) \leq 0,
\end{gathered}
$$

then by using the solution of the LMIs of (14), we consider the adaptive compensation input

$$
\psi\left(e_{y}, x_{t}, \hat{\theta}, t\right) \triangleq-\frac{1}{\left\|\Xi^{1 / 2} C e(t)\right\|^{2}} \omega\left(e_{y}, x_{t}, \hat{\theta}, t\right) \mathcal{T} e_{y}(t),
$$


where $\omega\left(e_{y}, x_{t}, \hat{\theta}, t\right)$ is the positive scalar function given by

$$
\begin{aligned}
\omega\left(e_{y}, x_{t}, \hat{\theta}, t\right) \triangleq & \frac{\left\|\Psi_{\gamma}^{1 / 2} e_{y}(t)\right\|^{4} \hat{\theta}^{2}(t)}{\left\|\Psi_{\gamma}^{1 / 2} e_{y}(t)\right\|^{2} \hat{\theta}(t)+\sigma(t)} \\
& +\frac{\left\|\Psi_{\epsilon}^{1 / 2} e_{y}(t)\right\|^{4}}{\left\|\Psi_{\epsilon}^{1 / 2} e_{y}(t)\right\|^{2}+\sigma(t)} \\
& +\frac{\gamma_{2}^{2}\left\|E x_{t}(t)\right\|^{4}}{\gamma_{2}\left\|E x_{t}(t)\right\|^{2}+\sigma(t)} .
\end{aligned}
$$

Besides, we introduce the following updating law for the adjustable parameter $\hat{\theta}(t) \in \mathbb{R}^{1}$ :

$$
\begin{aligned}
& \frac{d}{d t} \hat{\theta}(t) \triangleq-\frac{1}{2 \vartheta}\left\{\sigma(t)\left(\hat{\theta}(t)-\vartheta^{*}\right)\right. \\
&\left.-\left\|\Psi_{\gamma}^{1 / 2} e_{y}(t)\right\|^{2}+\sigma(t) \vartheta^{*}\right\} \\
&\left(=-\frac{1}{2 \vartheta}\left(\sigma(t)-\left\|\Psi_{\gamma}^{1 / 2} e_{y}(t)\right\|^{2}\right)\right) .
\end{aligned}
$$

Hereby asymptotical stability of the uncertain nonlinear error system of (13) is guaranteed. In (14), $\mathcal{Q} \in \mathbb{R}^{n \times n}$ is a symmetric positive definite matrix selected by designers, and $\sigma(t) \in \mathbb{R}^{1}$ in (16) is any positive uniform continuous and bounded function which satisfies

$$
\int_{t_{0}}^{t} \sigma(\tau) d \tau \leq \sigma^{*}<\infty
$$

where $t_{0}$ and $\sigma^{*}$ are an initial time and any positive constant, respectively.

Proof of Theorem 5. Firstly, we introduce the quadratic function

$$
\mathcal{V}(e, \hat{\theta}, t) \triangleq e^{T}(t) \operatorname{se}(t)+\vartheta\left(\hat{\theta}(t)-\vartheta^{*}\right)^{2}
$$

The time derivative of the quadratic function $\mathcal{V}(e, \hat{\theta}, t)$ can be written as

$$
\begin{aligned}
\frac{d}{d t} \mathcal{V}(e, \hat{\theta}, t)= & e^{T}(t)\left[H_{e}\left\{\& A_{K}\right\}\right] e(t) \\
+ & H_{e}\left\{e^{T}(t) \& B \Delta(t) E x(t)\right. \\
& \left.\quad+e^{T}(t) \& B \psi\left(e_{y}, x_{t}, \hat{\theta}, t\right)\right\} \\
& +H_{e}\left\{e^{T}(t) \& B\left(\xi(x, t)-\xi\left(x_{t}, t\right)\right)\right\} \\
& +2 \vartheta\left(\hat{\theta}(t)-\vartheta^{*}\right) \frac{d}{d t} \hat{\theta}(t) .
\end{aligned}
$$

Now, using Lemma 1 and the assumptions of (6) and (7), we can obtain the following relation for the time derivative of the quadratic function $\mathcal{V}(e, \hat{\theta}, t)$ :

$$
\begin{aligned}
& \frac{d}{d t} \mathcal{V}(e, \hat{\theta}, t) \leq e^{T}(t)\left[H_{e}\left\{\& A_{K}\right\}\right] e(t) \\
& +H_{e}\left\{e^{T}(t) \& B \Delta(t) E x(t)\right. \\
& \left.+e^{T}(t) \& B \psi\left(e_{y}, x_{t}, \hat{\theta}, t\right)\right\} \\
& +2 \chi^{*}\left\|B^{T} \varsigma e(t)\right\|\left\|\xi(x, t)-\xi\left(x_{t}, t\right)\right\| \\
& +2 \vartheta\left(\hat{\theta}(t)-\vartheta^{*}\right) \frac{d}{d t} \hat{\theta}(t) \\
& \leq e^{T}(t)\left[H_{e}\left\{\delta A_{K}\right\}\right] e(t) \\
& +H_{e}\left\{e^{T}(t) \& B \Delta(t) E e(t)\right. \\
& +e^{T}(t) \& B \Delta(t) E x_{t}(t) \\
& \left.+e^{T}(t) \& B \psi\left(e_{y}, x_{t}, \hat{\theta}, t\right)\right\} \\
& +2 \chi^{*}\left\|B^{T} s e(t)\right\|\|e(t)\| \\
& +2 \vartheta\left(\hat{\theta}(t)-\vartheta^{*}\right) \frac{d}{d t} \hat{\theta}(t) .
\end{aligned}
$$

Notice the fact that, for any positive constant $\mu$ and any vectors $a$ and $b$ with appropriate dimensions,

$$
2 a^{T} b \leq \mu a^{T} a+\frac{1}{\mu} b^{T} b
$$

Then some algebraic manipulations yield

$$
\begin{aligned}
\frac{d}{d t} \mathcal{V}(e, \hat{\theta}, t) \leq & e^{T}(t)\left[H_{e}\left\{\& A_{K}\right\}+\gamma_{1} E^{T} E+\epsilon\left(\chi^{*}\right)^{2} I_{n}\right] e(t) \\
& +H_{e}\left\{e^{T}(t) s C^{T} \mathcal{T}^{T} \psi\left(e_{y}, x_{t}, \hat{\theta}, t\right)\right\} \\
& +\gamma_{2} x_{t}^{T}(t) E^{T} E x_{t}(t) \\
& +\vartheta^{*}\left(\frac{1}{\gamma_{1}} e^{T}(t) s C^{T} \mathcal{T}^{T} \mathcal{T} C s e(t)\right. \\
& \left.+\frac{1}{\gamma_{2}} e^{T}(t) s C^{T} \mathcal{T}^{T} \mathcal{T} C \& e(t)\right) \\
& +\frac{1}{\epsilon} e^{T}(t) s C^{T} \mathcal{T}^{T} \mathcal{T} C s e(t) \\
& +2 \vartheta\left(\hat{\theta}(t)-\vartheta^{*}\right) \frac{d}{d t} \hat{\theta}(t) .
\end{aligned}
$$


Besides, we obtain the following inequality for the time derivative of the quadratic function $\mathcal{V}(e, \hat{\theta}, t)$,

$$
\begin{aligned}
\frac{d}{d t} \mathcal{V}(e, \hat{\theta}, t) \leq & e^{T}(t)\left[H_{e}\left\{\& A_{K}\right\}+\gamma_{1} E^{T} E+\epsilon\left(\chi^{*}\right)^{2} I_{n}\right] e(t) \\
& +H_{e}\left\{e^{T}(t) \& C^{T} \mathcal{T}^{T} \psi\left(e_{y}, x_{t}, \hat{\theta}, t\right)\right\} \\
& +\vartheta^{*} e^{T}(t) C^{T} \Psi_{\gamma} C e(t)+e^{T}(t) C^{T} \Psi_{\epsilon} C e(t) \\
& +\gamma_{2} x_{t}^{T}(t) E^{T} E x_{t}(t)+2 \vartheta\left(\hat{\theta}(t)-\vartheta^{*}\right) \frac{d}{d t} \hat{\theta}(t),
\end{aligned}
$$

because, by using Lemma 2 (Schur complement), one can see that the third LMI of (14) can be written as

$$
-C^{T} \Psi_{\gamma} C+\frac{1}{\gamma_{1}} \& C^{T} \mathcal{T}^{T} \mathcal{T} C \&+\frac{1}{\gamma_{2}} \& C^{T} \mathcal{T}^{T} \mathcal{T} C \& \leq 0,
$$

and fourth LMI of (14) is equivalent to the following matrix inequality:

$$
-C^{T} \Psi_{\epsilon} C+\frac{1}{\epsilon} \& C^{T} \mathcal{T}^{T} \mathcal{T} C \& \leq 0 .
$$

Using the first LMI and the second one of LMIs of (14) and introducing the adaptive compensation input of (15) and (16) and the updating law of (17), we have

$$
\begin{aligned}
& \frac{d}{d t} \mathcal{V}(e, \hat{\theta}, t) \leq-e^{T}(t) \mathcal{Q} e(t)+\vartheta^{*} e^{T}(t) C^{T} \Psi_{\gamma} C e(t) \\
&+e^{T}(t) C^{T} \Psi_{\epsilon} C e(t)+\gamma_{2} x_{t}^{T}(t) E^{T} E x_{t}(t) \\
&+H_{e}\left\{e^{T}(t) \& C^{T} \mathcal{T}^{T} \times\left(-\frac{1}{\left\|\Xi^{1 / 2} C e(t)\right\|^{2}}\right.\right. \\
&\left.\left.\times \omega\left(e_{y}, x_{t}, \hat{\theta}, t\right) \mathcal{T} e_{y}(t)\right)\right\} \\
&+ \\
&=-e^{T}(t)\left(\hat{\theta}(t)-\vartheta^{*}\right) \frac{d}{d t} \hat{\theta}(t)+\left\|\Psi_{\gamma}^{1 / 2} e_{y}(t)\right\|^{2} \hat{\theta}(t) \\
&- \sigma(t)\left(\hat{\theta}(t)-\vartheta^{*}\right)^{2}-\sigma(t) \vartheta^{*}\left(\hat{\theta}(t)-\vartheta^{*}\right) \\
&-\frac{\left\|\Psi_{\gamma}^{1 / 2} e_{y}(t)\right\|^{4} \hat{\theta}^{2}(t)}{\left\|\Psi_{\gamma}^{1 / 2} e_{y}(t)\right\|^{2} \hat{\theta}(t)+\sigma(t)} \\
&+\frac{\left\|\Psi_{\epsilon}^{1 / 2} e_{y}(t)\right\|^{2}}{\left\|\Psi_{\epsilon}^{1 / 2} e_{y}(t)\right\|^{2}+\sigma(t)} \sigma(t) \\
&+\frac{\gamma_{2}\left\|E x_{t}(t)\right\|^{2}}{\gamma_{2}\left\|E x_{t}(t)\right\|^{2}+\sigma(t)} \sigma(t) .
\end{aligned}
$$

In addition, utilizing the well-known inequality for any positive constants $\alpha$ and $\beta$,

$$
0 \leq \frac{\alpha \beta}{\alpha+\beta} \leq \alpha \quad \forall \alpha, \beta>0
$$

and some trivial manipulations give the relation

$$
\begin{aligned}
\frac{d}{d t} \mathcal{V}(e, \hat{\theta}, t) \leq & -e^{T}(t) \mathcal{Q} e(t)+3 \sigma(t)-\sigma(t)\left(\hat{\theta}(t)-\vartheta^{*}\right)^{2} \\
& -\sigma(t) \vartheta^{*}\left(\hat{\theta}(t)-\vartheta^{*}\right) .
\end{aligned}
$$

Namely, we have the following inequality:

$$
\frac{d}{d t} \mathcal{V}(e, \hat{\theta}, t) \leq-e^{T}(t) \mathcal{Q} e(t)+\varphi \sigma(t) .
$$

Here we have used the well-known inequality of (22), and $\varphi$ in (30) is a positive constant given by $\varphi \triangleq 3+\left(\vartheta^{*}\right)^{2}$. By letting $\zeta^{*} \triangleq \min \left\{\lambda_{\min }\{Q\}\right\}$, one can see that the inequality of (30) can be rewritten as

$$
\frac{d}{d t} \mathcal{V}(e, \hat{\theta}, t) \leq-\zeta^{*}\|e(t)\|^{2}+\varphi \sigma(t) .
$$

On the other hand, letting $e_{\hat{\theta}}(t)=\left(e^{T}(t) \hat{\theta}(t)\right)^{T}$, we see from the definition of the quadratic function $\mathcal{V}(e, \hat{\theta}, t)$ that there always exist two positive constants $\delta_{\min }$ and $\delta_{\max }$ such that, for any $t \geq t_{0}$,

$$
\xi^{-}\left(\left\|e_{\hat{\theta}}(t)\right\|\right) \leq \mathcal{V}(e, \hat{\theta}, t) \leq \xi^{+}\left(\left\|e_{\hat{\theta}}(t)\right\|\right),
$$

where $\xi^{-}\left(\left\|e_{\hat{\theta}}(t)\right\|\right)$ and $\xi^{+}\left(\left\|e_{\hat{\theta}}(t)\right\|\right)$ are given by

$$
\begin{aligned}
& \xi^{-}\left(\left\|e_{\hat{\theta}}(t)\right\|\right) \triangleq \delta_{\min }\left\|e_{\hat{\theta}}(t)\right\|^{2}, \\
& \xi^{+}\left(\left\|e_{\hat{\theta}}(t)\right\|\right) \triangleq \delta_{\max }\left\|e_{\hat{\theta}}(t)\right\|^{2} .
\end{aligned}
$$

It is obvious that any solution $e\left(t ; t_{0}, e\left(t_{0}\right)\right)$ of the uncertain nonlinear error system of (13) is continuous. In addition, it follows from (31) and (32) that, for any $t \geq t_{0}$, the relations of $0 \leq \xi^{-}\left(\left\|e_{\hat{\theta}}(t)\right\|\right) \leq \mathcal{V}(e, \hat{\theta}, t)$ and the following inequality holds:

$$
\begin{aligned}
\mathcal{V}(e, \hat{\theta}, t)= & \mathcal{V}\left(e, \hat{\theta}, t_{0}\right)+\int_{t_{0}}^{t} \frac{d}{d t} \mathcal{V}(e, \hat{\theta}, \tau) d \tau \\
\leq & \xi^{+}\left(\left\|e_{\hat{\theta}}\left(t_{0}\right)\right\|\right)-\int_{t_{0}}^{t} \xi^{*}(\|e(\tau)\|) d \tau \\
& +\varphi \int_{t_{0}}^{t} \sigma(\tau) d \tau .
\end{aligned}
$$

In (34), $\xi^{*}(\|e(t)\|)$ is defined as

$$
\xi^{*}(\|e(t)\|) \triangleq \zeta^{*}\|e(t)\|^{2} .
$$

Therefore, from (34) we can obtain the following two results. Firstly, taking the limit as $t$ approaches infinity on both sides of the inequality of (34), we have the inequality

$$
\begin{aligned}
0 \leq & \xi^{+}\left(\left\|e_{\hat{\theta}}\left(t_{0}\right)\right\|\right)-\lim _{t \rightarrow \infty} \int_{t_{0}}^{t} \xi^{*}(\|e(\tau)\|) d \tau \\
& +\varphi \lim _{t \rightarrow \infty} \int_{t_{0}}^{t} \sigma(\tau) d \tau .
\end{aligned}
$$


Thus one can see from (18) and (36) that

$$
\lim _{t \rightarrow \infty} \int_{t_{0}}^{t} \xi^{*}(\|e(\tau)\|) d \tau \leq \xi^{+}\left(\left\|e_{\hat{\theta}}\left(t_{0}\right)\right\|\right)+\varphi \sigma^{*} .
$$

On the other hand, from (34), we obtain

$$
0 \leq \xi^{-}\left(\left\|e_{\hat{\theta}}(t)\right\|\right) \leq \xi^{+}\left(\left\|e_{\hat{\theta}}\left(t_{0}\right)\right\|\right)+\varphi \int_{t_{0}}^{t} \sigma(\tau) d \tau .
$$

It follows from (18) and (38) that

$$
0 \leq \xi^{-}\left(\left\|e_{\hat{\theta}}(t)\right\|\right) \leq \xi^{+}\left(\left\|e_{\hat{\theta}}\left(t_{0}\right)\right\|\right)+\varphi \sigma^{*} .
$$

The relation of (39) implies that $e(t)$ is uniformly bounded. Since $e(t)$ has been shown to be continuous, it follows that $e(t)$ is uniformly continuous. Therefore, one can see that $\xi^{*}(\|e(t)\|)$ is also uniformly continuous. Thus applying Lemma 4 (Barbalat's lemma) to (37) yields

$$
\lim _{t \rightarrow \infty} \xi^{*}(\|e(t)\|)=\lim _{t \rightarrow \infty} \zeta^{*}\|e(t)\|=0 .
$$

Namely, asymptotical stability of the uncertain nonlinear error system of (13) is ensured. Thus the uncertain Lipschitz nonlinear system of (4) is also stable.

It follows that the result of the theorem is true. Thus the proof of Theorem 5 is completed.

Theorem 5 provides a sufficient condition for the existence of an adaptive gain robust output feedback controller for uncertain Lipschitz nonlinear system of (4). Next, we consider a special case. In this case, we deal with the uncertain Lipschitz nonlinear system described by

$$
\begin{gathered}
\frac{d}{d t} x(t)=(A+B \Delta(t) C) x(t)+B u(t)+\delta_{B}(x, t), \\
y(t)=C x(t) .
\end{gathered}
$$

In (41) the nonlinear term $\delta_{B}(x, t) \in \mathbb{R}^{n}$ satisfies

$$
\delta_{B}(x, t)=8^{-1} C^{T} \xi(x, t),
$$

where the matrix $\& \in \mathbb{R}^{n \times n}$ is symmetric positive definite and it is a solution of the following LMIs:

$$
\begin{gathered}
H_{e}\left\{\delta A_{K}\right\}+\epsilon\left(\chi^{*}\right)^{2} I_{n} \leq-\mathcal{Q}, \\
C^{T} \Xi C-H_{e}\left\{\delta C^{T} \mathcal{T}^{T} \mathcal{T} C\right\} \leq 0, \\
\left(\begin{array}{cc}
-C^{T} \Psi_{\gamma} C & 8 C^{T} \mathcal{T} \\
\star & -\gamma I_{m}
\end{array}\right) \leq 0 .
\end{gathered}
$$

In (43), $\mathcal{Q} \in \mathbb{R}^{n \times n}$ is a symmetric positive definite matrix selected by designers. Thus one can see from (6), (10)-(12), (42), and (43) that we have

$$
\begin{aligned}
& \frac{d}{d t} e(t)= A_{K} e(t)+B \Delta(t) C x(t) \\
&+s^{-1} C^{T}\left(\xi(x, t)-\xi\left(x_{t}, t\right)\right)+B \psi\left(e_{y}, x_{t}, \hat{\theta}, t\right) \\
& e_{y}(t)=C e(t) .
\end{aligned}
$$

Next theorem gives an LMI-based design method of an adaptive gain robust output feedback controller for this case.
Theorem 6. Consider the uncertain nonlinear error system of (44) with the adaptive compensation input $\psi\left(e_{y}, x_{t}, \hat{\theta}, t\right) \in$ $\mathbb{R}^{m}$.

If there exist symmetric positive definite matrices $\& \in$ $\mathbb{R}^{n \times n}, \Psi \in \mathbb{R}^{l \times l}$, and $\Xi \in \mathbb{R}^{l \times l}$ and the positive scalars $\gamma$ and $\epsilon$ satisfying the LMIs of (43), then by using the solution of the LMIs of (43), one considers the adaptive compensation input $\psi\left(e_{y}, x_{t}, \hat{\theta}, t\right) \in \mathbb{R}^{m}$ described as

$$
\begin{aligned}
& \psi\left(e_{y}, x_{t}, \hat{\theta}, t\right) \triangleq-\frac{1}{\left\|\Xi^{1 / 2} C e(t)\right\|^{2}} \omega_{B}\left(e_{y}, x_{t}, \hat{\theta}, t\right) \mathcal{T} e_{y}(t), \\
& \omega_{B}\left(e_{y}, x_{t}, \hat{\theta}, t\right) \triangleq \frac{\left\|\Psi_{\gamma}^{1 / 2} e_{y}(t)\right\|^{4} \hat{\theta}^{2}(t)}{\left\|\Psi_{\gamma}^{1 / 2} e_{y}(t)\right\|^{2} \hat{\theta}(t)+\sigma(t)} \\
& +\frac{\gamma^{2}\|y(t)\|^{4}}{\gamma\|y(t)\|^{2}+\sigma(t)} \\
& +\frac{\left\|\left(\epsilon^{-1}\right) e_{y}(t)\right\|^{4}}{\left\|\left(\epsilon^{-1}\right) e_{y}(t)\right\|^{2}+\sigma(t)}
\end{aligned}
$$

and the updating law of (17) for the adjustable parameter $\hat{\theta}(t) \in \mathbb{R}^{1}$. Hereby asymptotical stability of the uncertain error system with nonlinear terms of (4) is guaranteed. In (46), $\sigma(t) \in \mathbb{R}^{1}$ is any positive uniform continuous and bounded function satisfying (18).

Proof of Theorem 6. By using the symmetric positive definite matrix $\& \in \mathbb{R}^{n \times n}$, we consider the quadratic function $\mathcal{V}(e, \hat{\theta}, t)$ of (19). Then using the assumptions of (6) and (42) we have

$$
\begin{aligned}
\frac{d}{d t} \mathcal{V}(e, \hat{\theta}, t)= & e^{T}(t)\left[H_{e}\left\{\delta A_{K}\right\}\right] e(t) \\
& +H_{e}\left\{e^{T}(t) s C^{T} \mathcal{T}^{T} \Delta(t) C x(t)\right. \\
& \left.+e^{T}(t) \& C^{T} \mathcal{T}^{T} \psi\left(e_{y}, x_{t}, \hat{\theta}, t\right)\right\} \\
& +H_{e}\left\{e^{T}(t) C^{T}\left(\xi(x, t)-\xi\left(x_{t}, t\right)\right)\right\} \\
& +2 \vartheta\left(\hat{\theta}(t)-\vartheta^{*}\right) \frac{d}{d t} \hat{\theta}(t) \\
\leq & e^{T}(t)\left[H_{e}\left\{8 A_{K}\right\}\right] e(t) \\
& +H_{e}\left\{e^{T}(t) \& C^{T} \mathcal{T}^{T} \Delta(t) C x(t)\right\} \\
& +2 \chi^{*}\|C e(t)\|\|e(t)\| \\
& +H_{e}\left\{e^{T}(t) \& C^{T} \mathcal{T}^{T} \psi\left(e_{y}, x_{t}, \hat{\theta}, t\right)\right\} \\
& +2 \vartheta\left(\hat{\theta}(t)-\vartheta^{*}\right) \frac{d}{d t} \hat{\theta}(t) .
\end{aligned}
$$

In addition, applying the well-known inequality of (22) to the second term and the third one on the right-hand side of 
(47), we obtain the following relation for the time derivative of the quadratic function $\mathcal{V}(e, \hat{\theta}, t)$ :

$$
\begin{aligned}
\frac{d}{d t} \mathcal{V}(e, \hat{\theta}, t) \leq & e^{T}(t)\left[H_{e}\left\{\& A_{K}\right\}+\epsilon\left(\chi^{*}\right)^{2} I_{n}\right] e(t) \\
& +\frac{\vartheta^{*}}{\gamma} e^{T}(t) \& C^{T} \mathcal{T}^{T} \mathcal{T} C \& e(t) \\
& +\gamma y^{T}(t) y(t)+\frac{1}{\epsilon} e_{y}^{T}(t) e_{y}(t) \\
& +H_{e}\left\{e^{T}(t) \& C^{T} \mathcal{T}^{T} \psi\left(e_{y}, x_{t}, \hat{\theta}, t\right)\right\} \\
& +2 \vartheta\left(\hat{\theta}(t)-\vartheta^{*}\right) \frac{d}{d t} \hat{\theta}(t) .
\end{aligned}
$$

Now, one can see from (17), (43), (45), and (46) that if the following inequality holds, then the condition of (48) is also satisfied

$$
\begin{aligned}
\frac{d}{d t} \mathcal{V}(e, \hat{\theta}, t) \leq & -e^{T}(t) \mathcal{Q} e(t)+\frac{\vartheta^{*}}{\gamma} e^{T}(t) C^{T} \Psi_{\gamma} C e(t) \\
& +\gamma y^{T}(t) y(t)+\frac{1}{\epsilon} e_{y}^{T}(t) e_{y}(t) \\
& +H_{e}\left\{e^{T}(t) 8 C^{T} \mathcal{T}^{T} \psi\left(e_{y}, x_{t}, \hat{\theta}, t\right)\right\} \\
& +2 \vartheta\left(\hat{\theta}(t)-\vartheta^{*}\right) \frac{d}{d t} \hat{\theta}(t) \\
\leq & -e^{T}(t) \mathcal{Q} e(t)+\left\|\Psi_{\gamma}^{1 / 2} e_{y}(t)\right\|^{2} \hat{\theta}(t) \\
& -\sigma(t)\left(\hat{\theta}(t)-\vartheta^{*}\right)^{2}-\sigma(t) \vartheta\left(\hat{\theta}(t)-\vartheta^{*}\right) \\
& -\frac{\left\|\Psi_{\gamma}^{1 / 2} e_{y}(t)\right\|^{4} \hat{\theta}^{2}(t)}{\left\|\Psi_{\gamma}^{1 / 2} e_{y}(t)\right\|^{2} \hat{\theta}(t)+\sigma(t)} \\
& +\frac{\gamma\|y(t)\|^{2}}{\gamma\|y(t)\|^{2}+\sigma(t)} \sigma(t) \\
& +\frac{\left\|\left(\epsilon^{-1}\right) e_{y}(t)\right\|^{2}}{\left\|\left(\epsilon^{-1}\right) e_{y}(t)\right\|^{2}+\sigma(t)} \sigma(t) .
\end{aligned}
$$

Besides, from (49) we obtain the inequality of (30). Therefore, one can see from Proof of Theorem 5 that the rest of proof of Theorem 6 is straightforward.

Remark 7. The proposed control scheme is adaptable when some assumptions are satisfied, and in cases where only the output signal of the system is available, the proposed scheme can be used widely. In addition, the proposed controller is more effective for systems with large uncertainties and Lipschitz constants. Note that the adjustable parameter $\hat{\theta}(t) \in \mathbb{R}^{1}$ is not an estimate of the unknown bound $\vartheta^{*}$.

\section{Numerical Examples}

In order to demonstrate the efficiency of the proposed control scheme, we have run a simple example. The control problem considered here is not necessary practical. However, the simulation results stated below illustrate the distinct feature of the proposed output feedback controller.

Consider the uncertain Lipschitz nonlinear system described by the following state equation

$$
\begin{aligned}
\frac{d}{d t} x(t)= & \left(\begin{array}{ccc}
-2.0 & 0.0 & -6.0 \\
0.0 & 1.0 & 1.0 \\
3.0 & 0.0 & -7.0
\end{array}\right) x(t) \\
& +\left(\begin{array}{l}
2.0 \\
1.0 \\
0.0
\end{array}\right) \Delta(t)\left(\begin{array}{lll}
1.0 & 0.0 & 1.0 \\
0.0 & 3.0 & 1.0
\end{array}\right) x(t) \\
& +\left(\begin{array}{l}
2.0 \\
1.0 \\
0.0
\end{array}\right) u(t)+\left(\begin{array}{l}
2.0 \\
1.0 \\
0.0
\end{array}\right) \xi(x, t), \\
y(t) & =\left(\begin{array}{lll}
1.0 & 0.0 & 0.0 \\
0.0 & 1.0 & 0.0
\end{array}\right) x(t),
\end{aligned}
$$

that is, $\mathcal{T}=\left(\begin{array}{ll}2.0 & 1.0\end{array}\right)$. In this example we assume that the function $\xi(x, t)$ and the positive scalar $\chi^{*} \in \mathbb{R}^{1}$ in (5) are given by $\xi(x, t)=\sqrt{3.0} \times \sin \left(x_{2}(t)\right)$ and $\chi^{*}=\sqrt{3.0}$, respectively.

Firstly by adopting the similar way to the standard linear quadratic control problem, we consider designing the fixed gain matrix $\mathcal{K}_{\mathscr{Q} Q} \in \mathbb{R}^{2 \times 3}$. Thus selecting the design parameters $\mathcal{Q}_{t} \in \mathbb{R}^{3 \times 3}$ and $\mathcal{R}_{t} \in \mathbb{R}^{1}$ such that $\mathcal{Q}_{t}=1.0 \times I_{3}$ and $\mathcal{R}_{t}=1.0 \times 10^{1}$, respectively, and solving the algebraic Riccati equation $H_{e}\left\{A^{T} \mathcal{X}_{t}\right\}-\mathcal{X}_{t} B \mathcal{R}_{t}^{-1} B^{T} \mathcal{X}_{t}+\mathcal{Q}_{t}=0$, we obtain

$$
\begin{aligned}
& X_{t}=\left(\begin{array}{ccc}
2.23662 \times 10^{-1} & 1.04459 & 1.95383 \times 10^{-2} \\
\star & 1.6170145 \times 10^{1} & 1.25624 \\
\star & \star & 2.22160 \times 10^{-1}
\end{array}\right), \\
& K_{\mathscr{L} Q}=\left(-1.49191 \times 10^{-1}-1.82593-1.29532 \times 10^{-1}\right) \text {. }
\end{aligned}
$$

Next we design an output feedback gain matrix $K \in$ $\mathbb{R}^{1 \times 2}$ by using the the nominal system of (9). We select the design parameter $\alpha \in \mathbb{R}^{1}$ such as $\alpha=5.0$, then by applying the LMI-based design algorithm (see [29] and the Appendix section in [15]), we obtain the following solutions for LMI problems in Appendix in [15] and the following gain matrices, respectively:

$$
\begin{aligned}
& X=\left(\begin{array}{ccc}
1.26046 \times 10^{3} & 1.25379 \times 10^{2} & 3.19323 \times 10^{2} \\
\star & 8.97329 \times 10^{1} & -1.13357 \times 10^{2} \\
\star & \star & 5.45874 \times 10^{2}
\end{array}\right) \text {, } \\
& y=\left(1.02905 \times 10^{3}-5.78548 \times 10^{2}-4.11077 \times 10^{2}\right) \text {, }
\end{aligned}
$$




$$
\begin{gathered}
\mathcal{P}=\left(\begin{array}{ccc}
1.34029 \times 10^{1} & -4.46413 \times 10^{1} & -2.31136 \\
\star & 1.74644 \times 10^{2} & 4.12700 \\
\star & \star & 1.03904
\end{array}\right), \\
\rho=4.38355 \times 10^{4} \\
K_{\mathrm{sf}}=\left(\begin{array}{lll}
2.18703 & -1.63634 \times 10^{1} & -5.43048
\end{array}\right) \\
K=\left(3.17745 \times 10^{-1}-1.20809 \times 10^{1}\right)
\end{gathered}
$$

Now, we use Theorem 5 to design the proposed adaptive gain robust output feedback controller; that is, we solve the LMIs of (14). By selecting the symmetric positive definite matrix $\mathcal{Q} \in \mathbb{R}^{3 \times 3}$ such as $\mathcal{Q}=0.1 \times I_{3}$, we have

$$
\begin{aligned}
& \delta=\left(\begin{array}{ccc}
1.67330 & -1.49618 & -6.50900 \times 10^{-3} \\
\star & 4.17725 & 1.48476 \times 10^{-1} \\
\star & \star & 6.58185
\end{array}\right) \\
& \Xi=\left(\begin{array}{cc}
4.30003 & 2.32356 \\
\star & 2.04206
\end{array}\right), \\
& \Psi_{\gamma}=\left(\begin{array}{cc}
1.07015 & 8.119016 \times 10^{-1} \\
\star & 1.00293
\end{array}\right) \text {, } \\
& \Psi_{\epsilon}=\left(\begin{array}{cc}
9.56125 & 7.12983 \\
\star & 9.13154
\end{array}\right) \\
& \gamma_{1}=7.14302, \quad \gamma_{2}=7.57592 \text {, } \\
& \epsilon=9.55061 \text {. }
\end{aligned}
$$

In this example, we consider the following two cases for the unknown parameter $\Delta(t) \in \mathbb{R}^{1 \times 2}$ and its unknown upper bound $\vartheta^{*}$ in $(5)$ :

Case (1):

$$
\begin{aligned}
& \vartheta^{*}=5.0 \\
& \Delta(t)=\vartheta^{*} \times\left(\begin{array}{ll}
8.113249 & -5.56040
\end{array}\right) \times 10^{-1} .
\end{aligned}
$$

Case (2):

$$
\begin{gathered}
\vartheta^{*}=5.0 \times 10^{-1} \\
\Delta(t)=\vartheta^{*} \times(\sin (10 \pi t) \cos (10 \pi t)) .
\end{gathered}
$$

Furthermore, initial values for the uncertain system of (50), its target model, and the adjustable parameter $\hat{\theta}(t)$ are selected as $x(0)=\left(\begin{array}{lll}1.5 & 2.0 & -4.5\end{array}\right)^{T}, x_{t}(0)=$ $\left(\begin{array}{lll}2.0 & 2.0-5.0\end{array}\right)^{T}$, and $\hat{\theta}(0)=1.0$, respectively. Besides, we choose $\sigma(t) \in \mathbb{R}^{+}$in (16) and the design parameter $\vartheta \in \mathbb{R}^{1}$ in $(17)$ such as $\sigma(t)=5.0 \times 10^{4} \times \exp \left(-1.0 \times 10^{-4} t\right)$ and $\vartheta=2.5 \times 10^{3}$, respectively.

The results of the simulation of this example are depicted in Figures 1, 2, 3, 4, and 5. In these figures, the time histories

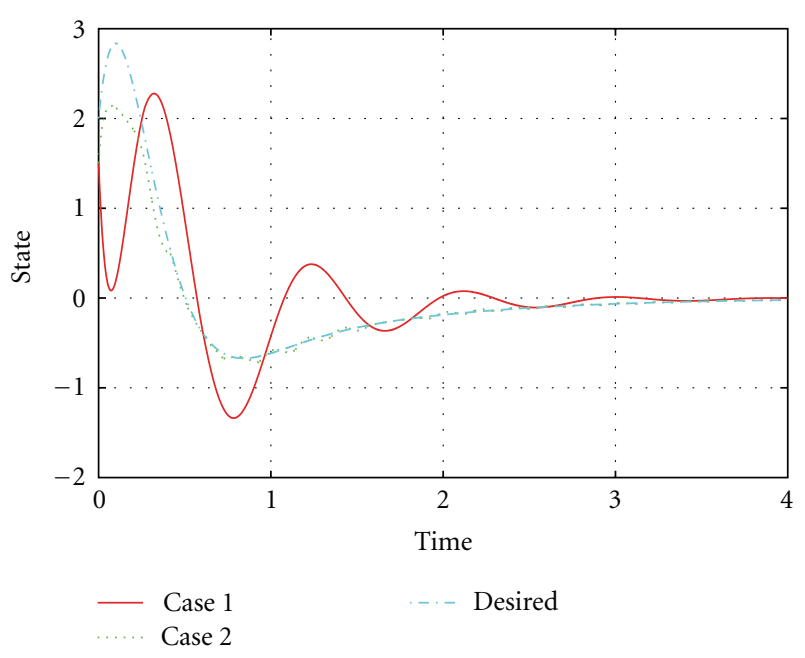

Figure 1: Time histories of the state variable $x_{1}(t)$.

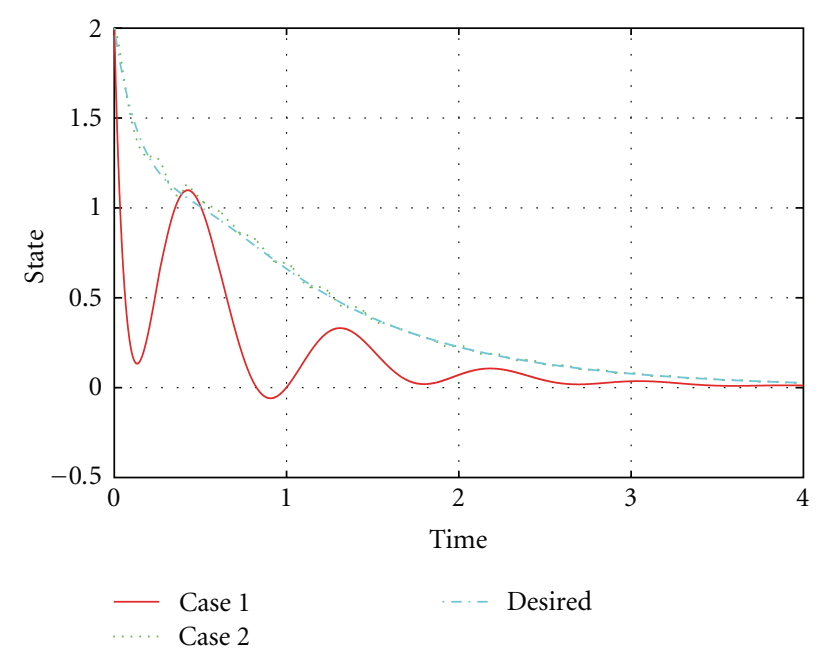

FIgURE 2: Time histories of the state variable $x_{2}(t)$.

of the state variables $x_{1}(t), x_{2}(t)$, and $x_{3}(t)$, the control input $u(t)$, and the adjustable parameter $\theta(t) \in \mathbb{R}^{1}$ are shown. "Desired" in these figures represents the trajectories for the state variables and the control input generated by the target model; that is, "Desired" for state variables shows a desirable response for the uncertain nonlinear systems.

From Figures 1, 2, 3, and 4, we find that the proposed adaptive gain robust output feedback controller stabilizes the controlled system of (50) in spite of plant uncertainties and nonlinearities. Besides, one can see that the proposed adaptive gain robust output feedback controller achieves good transient performance and the proposed control input is tuned by the measurable signals and the adjustable parameter. In particular the time responses of the uncertain nonlinear system of (50) for Case (2) close to the trajectory of the target model and the control input also achieves satisfactory trajectory. This result shows that the proposed adaptive robust output feedback controller reflects the effect of uncertainties and nonlinearities as online information. 


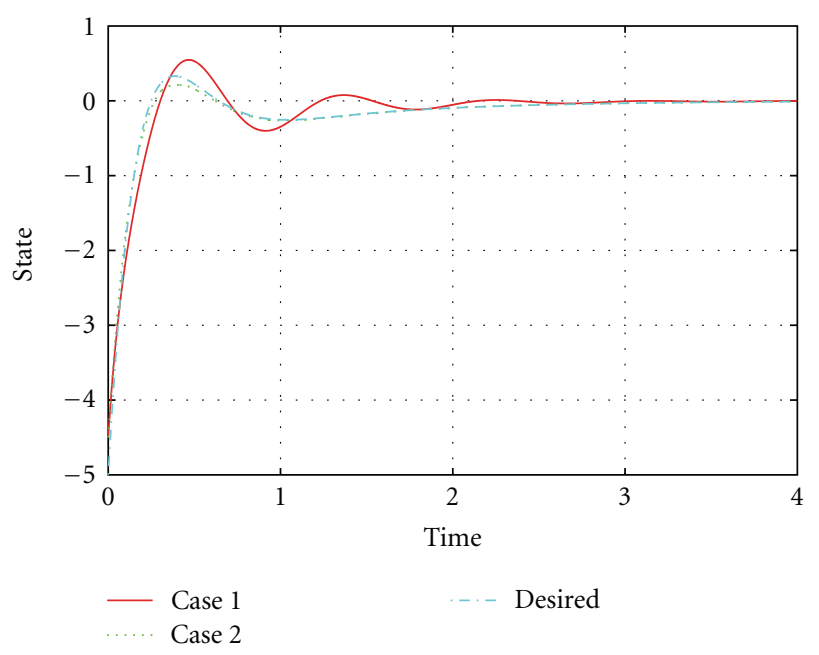

Figure 3: Time histories of the state variable $x_{3}(t)$.

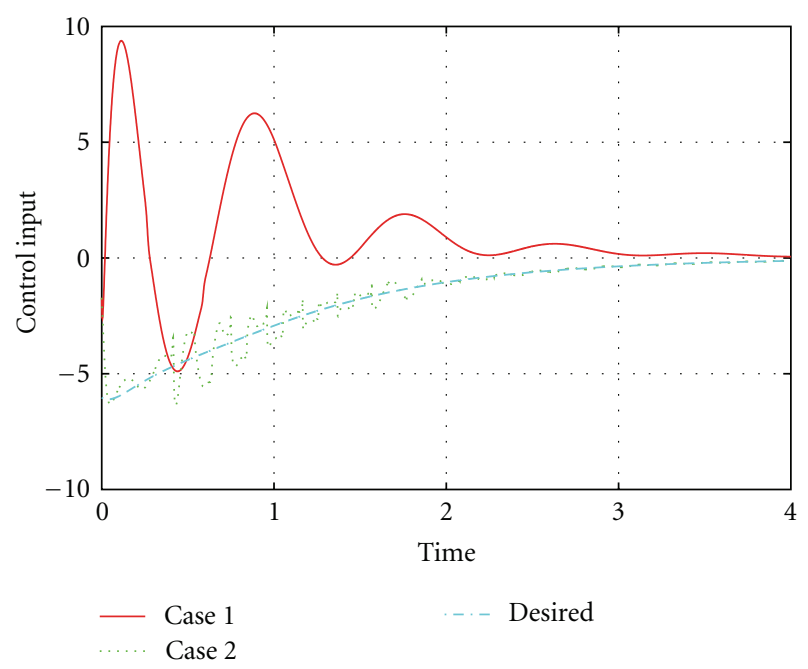

Figure 4: Time histories of the control input $u(t)$.

Furthermore, we can see from Figure 5 that the parameter $\hat{\theta}(t)$ is tuned by the updating law and is not an estimate of the upper bound $\vartheta^{*}$.

\section{Conclusions}

In this paper, we have proposed an adaptive gain robust output feedback controller for a class of uncertain Lipschitz nonlinear system of which upper bounds are unknown. For the uncertain Lipschitz nonlinear system, we have shown that the proposed adaptive gain robust output feedback controller can be obtained by solving LMIs. Besides, by numerical simulations, the effectiveness of the proposed adaptive robust controller has been presented. One can see that the crucial difference between the existing results [24] and our new one is that information is not required on the upper bound of the unknown parameter $\Delta(t)$ in the system matrix. The proposed controller design method is adaptable when some assumptions are satisfied and in cases where only the output

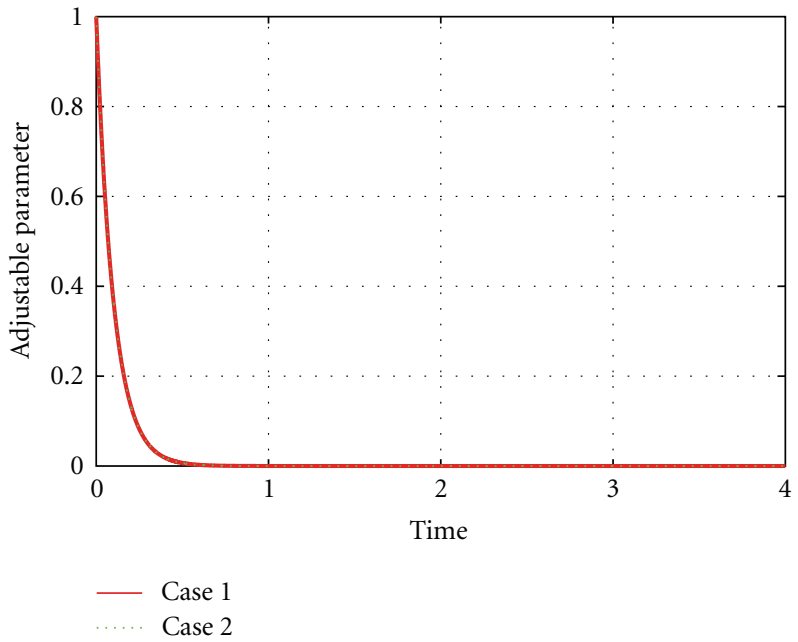

Figure 5: Time histories of the adjustable parameter $\hat{\theta}(t)$.

signal of the system is available. Namely, if for uncertain Lipschitz nonlinear systems which satisfy the assumptions for the system parameters, the LMIs of (14) are feasible, then the proposed adaptive gain robust output feedback controller is applicable.

The future research subjects are extension of the proposed adaptive gain robust output feedback controller synthesis to such a broad class of systems as uncertain time-delay systems and uncertain large-scale interconnected systems. Furthermore in future work, we will examine the assumption of (7).

\section{Acknowledgment}

The authors would like to thank CAE Solutions Corp. for providing its support in conducting this study. Additionally, the authors would like to thank Professor Zhiyong Chen with the University of Newcastle and the anonymous reviewers for their valuable and helpful comments that greatly contributed to this paper.

\section{References}

[1] K. Zhou, Essentials of Robust Control, Prentice Hall, Upper Saddle River, NJ, USA, 1998.

[2] B. R. Barmish, "Stabilization of uncertain systems via linear control," IEEE Transactions on Automatic Control, vol. 8, no. 8, pp. 848-850, 1983.

[3] I. R. Petersen and C. V. Hollot, "A riccati equation approach to the stabilization of uncertain linear systems," Automatica, vol. 22, no. 4, pp. 397-411, 1986.

[4] J. C. Doyle, K. Glover, P. P. Khargonekar, and B. A. Francis, "State-space solutions to standard $\mathrm{H}_{2}$ and $\mathrm{H}_{\infty}$ control problems," IEEE Transactions on Automatic Control, vol. 34, no. 8, pp. 831-847, 1989.

[5] S. Yamamoto and K. Yamauchi, "A design method of adaptive control systems by a time-varying parameter of robust stabilizing state feedback," Transactions of the Institute of Systems, Control and Information Engineers, vol. 12, no. 6, pp. 319-325, 1999 (Japanese). 
[6] M. Maki and K. Hagino, "Robust control with adaptation mechanism for improving transient behaviour," International Journal of Control, vol. 72, no. 13, pp. 1218-1226, 1999.

[7] H. Oya and K. Hagino, "Robust control with adaptive compensation input for linear uncertain systems," Transactions on Fundamentals of Electronics, Communications and Computer Sciences, vol. 86, no. 6, pp. 1517-1524, 2003.

[8] H. Oya and K. Hagino, "Adaptive robust control scheme for linear systems with structured uncertainties," Transactions on Fundamentals of Electronics, Communications and Computer Sciences, vol. 87, no. 8, pp. 2168-2173, 2004.

[9] J. R. Petersen, "A riccati equation approach to the design of stabilizing controllers and observers for a class of uncertain linear systems," IEEE Transactions on Automatic Control, vol. 30, no. 9, pp. 904-907, 1985.

[10] H. Oya and K. Hagino, "Observer-based robust control giving consideration to transient behaviour for linear systems with structured uncertainties," International Journal of Control, vol. 75, no. 15, pp. 1231-1240, 2002.

[11] S. O. R. Moheimani and I. R. Petersen, "Optimal guaranteed cost control of uncertain systems via static and dynamic output feedback," Automatica, vol. 32, no. 4, pp. 575-579, 1996.

[12] J. C. Geromel, C. C. De Souza, and R. E. Skelton, "LMI numerical solution for output feedbadck stabilization," in Proceedings of the American Control Conference, pp. 40-44, Baltimore, Md, USA, 1994.

[13] T. Iwasaki, R. E. Skelton, and J. C. Geromel, "Linear quadratic suboptimal control with static output feedback," Systems and Control Letters, vol. 23, no. 6, pp. 421-430, 1994.

[14] M. Matsuoka and K. Hagino, "A robust control design via a variable gain controller using an observer without information on the upper bound of uncertainties," in Proceedings of the 10th International Conference on Information System Analysis and Synthesis, pp. 324-329, 2004.

[15] H. Oya, K. Hagino, and S. Kayo, "Synthesis of adaptive robust output feedback controllers for a class of uncertain linear systems," in Proceedings of the 47th IEEE Conference on Decision and Control (CDC '08), pp. 995-1000, Cancun, Mexico, December 2008.

[16] F. Mazenc, L. Praly, and W. P. Dayawansa, "Global stabilization by output feedback: examples and counterexamples," Systems and Control Letters, vol. 23, no. 2, pp. 119-125, 1994.

[17] A. N. Atassi and H. K. Khalil, "A separation principle for the stabilization of a class of nonlinear systems," IEEE Transactions on Automatic Control, vol. 44, no. 9, pp. 1672-1687, 1999.

[18] L. Praly, "Asymptotic stabilization via output feedback for lower triangular systems with output dependent incremental rate," in Proceedings of the 40th IEEE Conference on Decision and Control (CDC '01), pp. 3808-3813, Orlando, Fla, USA, December 2001.

[19] C. Qian, "A homogeneous domination approach for global output feedback stabilization of a class of nonlinear systems," in Proceedings of the American Control Conference (ACC '05), pp. 4708-4715, Portland, Ore, USA, June 2005.

[20] J. Polendo and C. Qian, "A generalized homogeneous domination approach for global stabilization of inherently nonlinear systems via output feedback," International Journal of Robust and Nonlinear Control, vol. 17, no. 7, pp. 605-629, 2007.

[21] J. Li and C. Qian, "Global finite-time stabilization by dynamic output feedback for a class of continuous nonlinear systems," IEEE Transactions on Automatic Control, vol. 51, no. 5, pp. 879-884, 2006.
[22] J. Tsinias, "Backstepping design for time-varying nonlinear systems with unknown parameters," Systems and Control Letters, vol. 39, no. 4, pp. 219-227, 2000.

[23] H. L. Choi and J. T. Lim, "Output feedback stabilization for a class of Lipschitz nonlinear systems," Transactions on Fundamentals of Electronics, Communications and Computer Sciences, vol. 88, no. 2, pp. 602-605, 2005.

[24] H. Oya and K. Hagino, "Synthesis of variable gain robust output feedback controllers for a class of uncertain lipschitz nonlinear systems," in Proceedings of the 9th International Conference on Control \& Automation, pp. 698-703, Santiago, Chile, December 2011.

[25] F. R. Gantmacher, The Theory of Matrices, vol. 1, Chelsea Publishing Company, New York, NY, USA, 1960.

[26] S. Boyd, L. El Ghaoui, E. Feron, and V. Balakrishnan, Linear Matrix Inequalities in System and Control Theory, SIAM Studies in Applied Mathmatics, 1994.

[27] H. K. Khalil, Nonlinear Systems, Prentice Hall, 3rd edition, 2002.

[28] V. Kučera and C. E. De Souza, "A necessary and sufficient condition for output feedback stabilizability," Automatica, vol. 31, no. 9, pp. 1357-1359, 1995.

[29] R. E. Benton and D. Smith, "Non-iterative LMI-based algorithm for robust static-output-feedback stabilization," International Journal of Control, vol. 72, no. 14, pp. 1322-1330, 1999. 

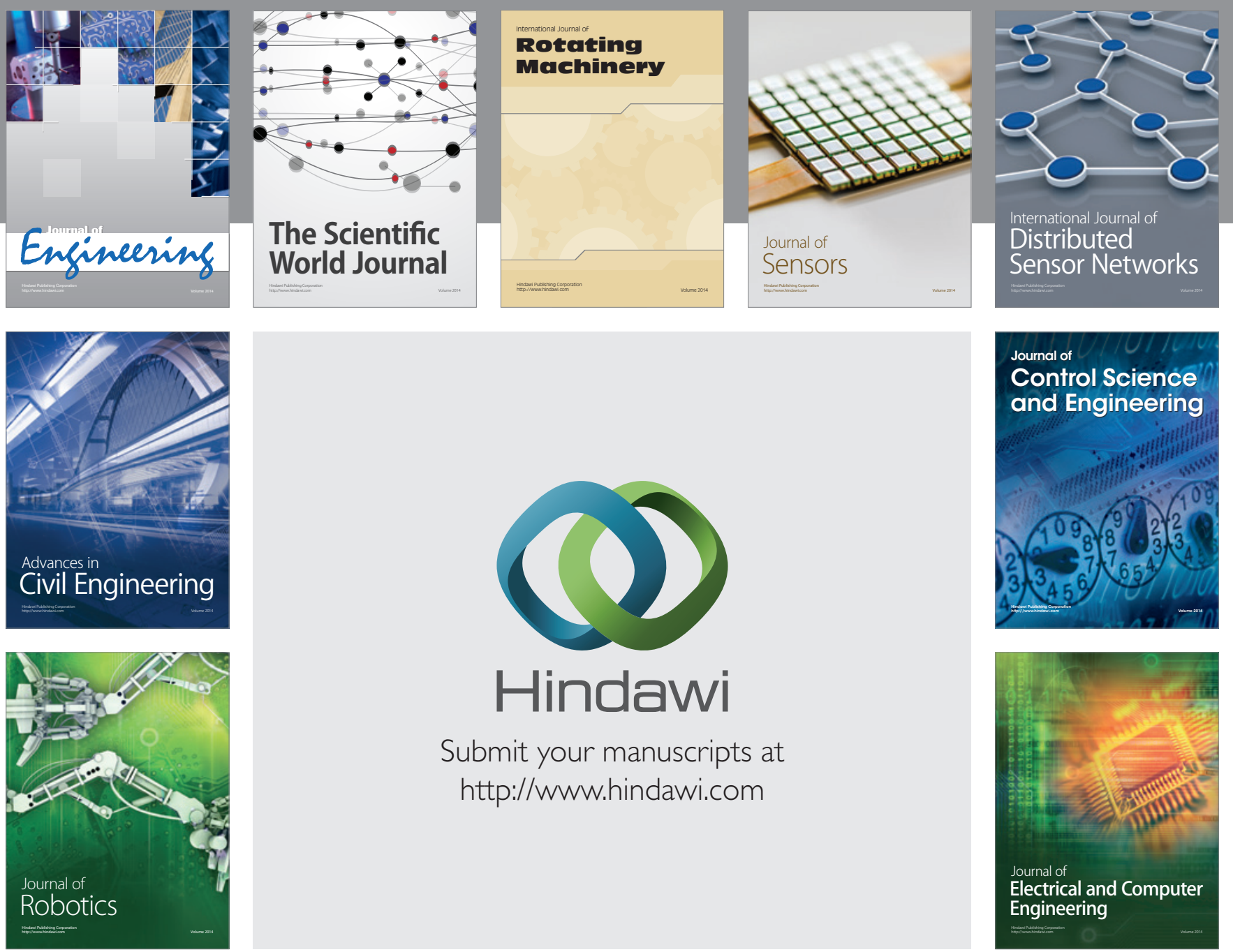

Submit your manuscripts at

http://www.hindawi.com
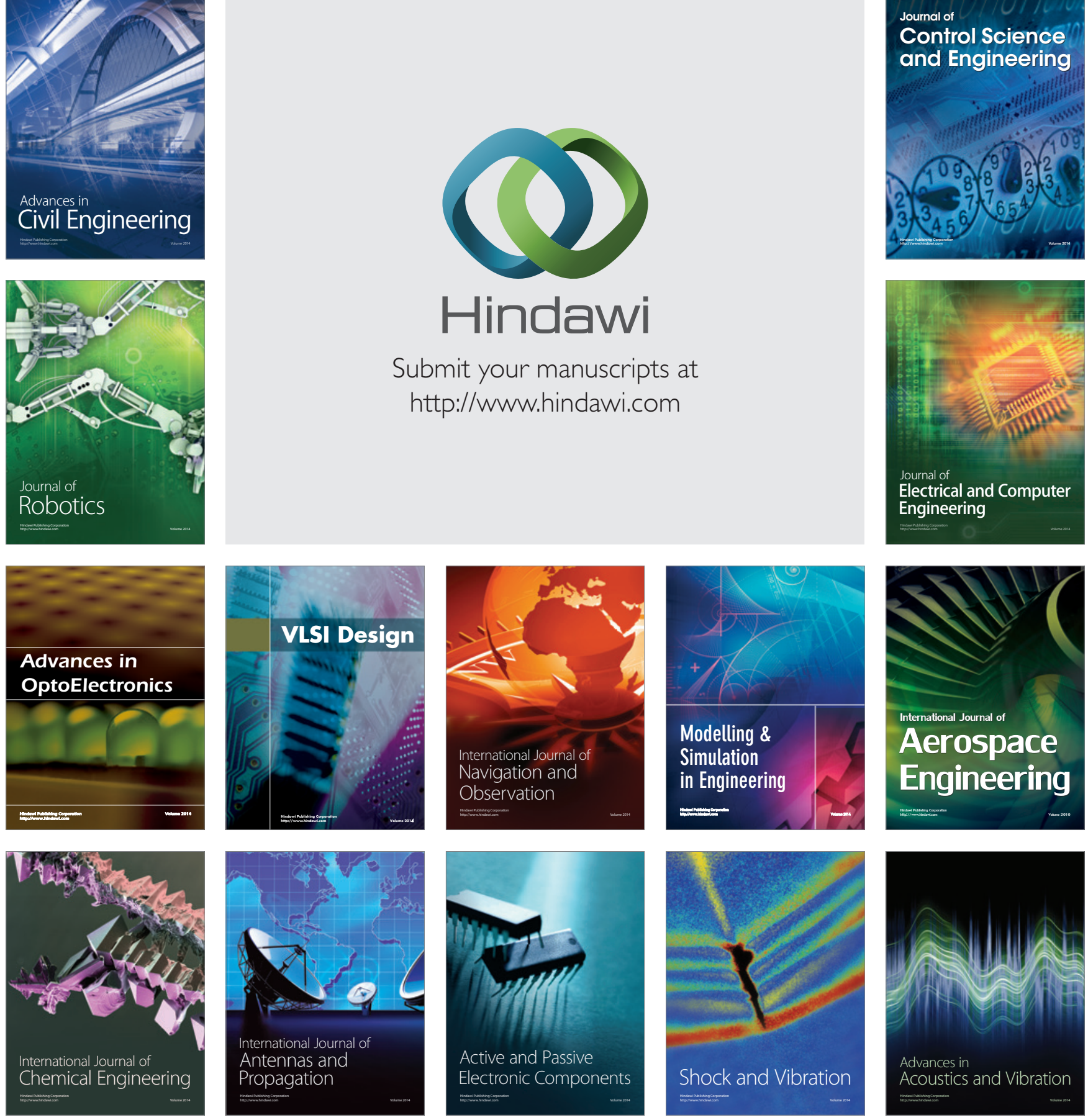